\title{
Mucopolysaccharidosis type IV as a cause of mitral stenosis in an adult
}

\author{
M A IRELAND, D B ROWLANDS \\ From the Department of Cardiovascular Medicine, East Birmingham Hospital, Birmingham
}

SUMMARY We report a case of mucopolysaccharidosis type IV causing severe mitral stenosis in a 48-year-old woman.

The mucopolysaccharidoses are a group of disorders characterised by defective enzymatic activity leading to storage of incompletely degraded glycosaminoglycans. There are six distinct enzymatic defects dividing the mucopolysaccharidoses into six separate types; some of these are further subdivided on the basis of the clinical features. As a group patients with mucopolysaccharidosis tend to have coarse facies, short stature, and skeletal dysplasia. Each type has distinct clinical features accompanying the general features which has resulted in a plethora of eponymous syndromes. Mitral stenosis has been described as a consequence of cardiac involvement in mucopolysaccharidosis type I (The HurlerScheie syndrome) and in type VI (the MaroteauxLamy syndrome). ${ }^{1}$

Mucopolysaccharidosis type IV (MorquioBrailsford syndrome) is caused by a deficiency of $\mathrm{N}$-acetylhexosaminidase-6- $\mathrm{SO}_{4}$ sulphatase resulting in excessive excretion of keratan sulphate in the urine. Typically, the patients are dwarfed, with thoracolumbar gibbus and a protuberant sternum. The joints of the hands are lax and the intellect is normal. Corneal clouding and deafness develop in later childhood. Aortic regurgitation occurring in early adult life is well recognised, ${ }^{2}$ though other valvular involvement has hitherto been unreported. We report a case of an adult with mucopolysaccharidoses type IV who had severe mitral stenosis and histological evidence of involvement of the other three valves.

\section{Case report}

The patient, aged 48 years, was admitted to hospital as an emergency in November 1977 with acute dyspnoea. She had the typical features of mucopolysaccharidosis type IV, being a dwarf of $100 \mathrm{~cm}$ in height, with characteristic facies-a broad mouth, prominent maxilla, short nose, and widely spaced teeth. Examination of the eyes disclosed bilateral corneal opacification resulting in blindness. There was severe protuberance of the abdomen, with shortening of the trunk and a slight pectus carinatum. Both upper and lower limbs were also shortened but appeared to be of the correct proportions; there was, however, ulnar deviation of the hands at the metacarpophalangeal joints and bilateral genu valgum. Spastic paraparesis was also present. On admission she was cyanosed, tachypnoeic, and had bilateral crepitations diagnosed as being the result of pulmonary oedema secondary to left ventricular failure. Treatment with fluid restriction, bed rest, and frusemide resulted in prompt improvement and she was discharged on a combined preparation of chlorothiazide and amiloride.

The diagnosis of mucopolysaccharidosis type IV was confirmed biochemically, cellulose acetate membrane electrophoresis of the urine showing the typical "C pattern". 3 The urinary glycosaminoglycan level was grossly raised at $168 \mathrm{mg} / \mathrm{g}$ creatinine (normal $<15 \mathrm{mg} / \mathrm{g}$ ) as was the urinary uronic acid level, at $36 \mathrm{mg} / \mathrm{g}$ creatinine (normal $<10 \mathrm{mg} / \mathrm{g}$ ).

She was readmitted in October 1978 with an exacerbation of breathlessness and she complained of deterioration in her exercise tolerance. Physical examination disclosed signs of pulmonary oedema and an apical systolic murmur radiating to the axilla. An electrocardiogram showed sinus rhythm with left atrial hypertrophy. Echocardiography was performed with some difficulty because of the thoracic deformity and measurements neither of the left ventricular dimension nor of the rate of left ventricular filling were possible. The mitral valve was abnormally thickened with reduced mobility. The diastolic closure rate was less than $15 \mathrm{~mm} / \mathrm{s}$, indicating severe mitral stenosis. The aortic valve 
was not visualised, but the left atrium appeared enlarged.

Similar antifailure treatment with the addition of digoxin was given with good effect. In view of the diagnosis of severe mitral stenosis further investigation was discussed with the patient and her mother. Both preferred to continue with medical treatment in the hope of avoiding surgery. She was discharged on digoxin, bumetanide and potassium in a combined tablet, and warfarin.

During the following eight months she became progressively more dyspnoeic with effort and was readmitted to hospital in June 1979 with another episode of acute pulmonary oedema. On this occasion she failed to improve with medical treatment and died three days after admission.

At necropsy the heart was enlarged, weighing $254 \mathrm{~g}$. The left ventricular wall was $1.5 \mathrm{~cm}$ thick and the right ventricular wall $0.6 \mathrm{~cm}$ thick, both chambers showing concentric hypertrophy. The mitral valve measured $6.5 \mathrm{~cm}$ in circumference and showed severe irregularity of both cusps, the anterior more than the posterior. The cusps were thickened and rolled, showing numerous distended capillaries on both surfaces, and there was shortening and thickening of all chordae, especially those to the anterior cusp (Fig. 1). The aortic valve measured $7.5 \mathrm{~cm}$ and there was thickening of the valve cusps throughout with some fusion of the commissures. The tricuspid valve measured $9.0 \mathrm{~cm}$

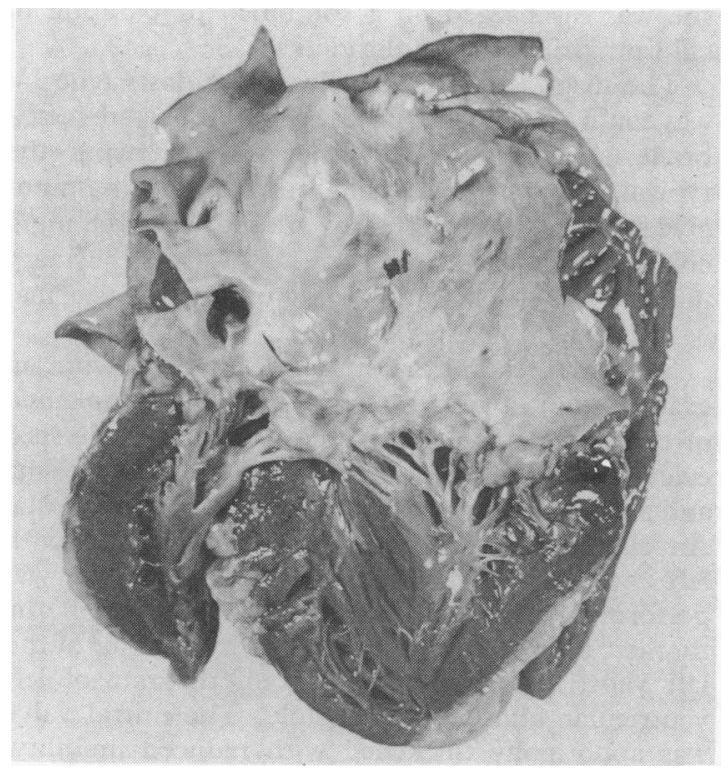

Fig. 1 The mitral valve cusps are thickened with rolled edges. The chordae are also thickened. in circumference and the valve cusps again were $\stackrel{c}{\mathrm{C}}$ irregularly thickened with some thickening of the $\overrightarrow{\vec{F}}$ chordae to the middle cusp (Fig. 2). The pulmonary valve measured $8.0 \mathrm{~cm}$ in circumference; several fenestrations were seen in the cusps and there was $\frac{\bar{D}}{\bar{D}}$ ridge-like thickening at the base of the cusps with $\overrightarrow{\widetilde{D}}$ slight commissural fusion. The coronary arteries $\cong$ showed minimal atheroma and were widely patent. के There was no evidence of pericardial thickening. $\overrightarrow{0}$ Histological examination of all four valves confirmed $\overrightarrow{\mid}$ infiltration with mucopolysaccharide when stained $\vec{\omega}$ with Aician blue. ${ }^{4}$

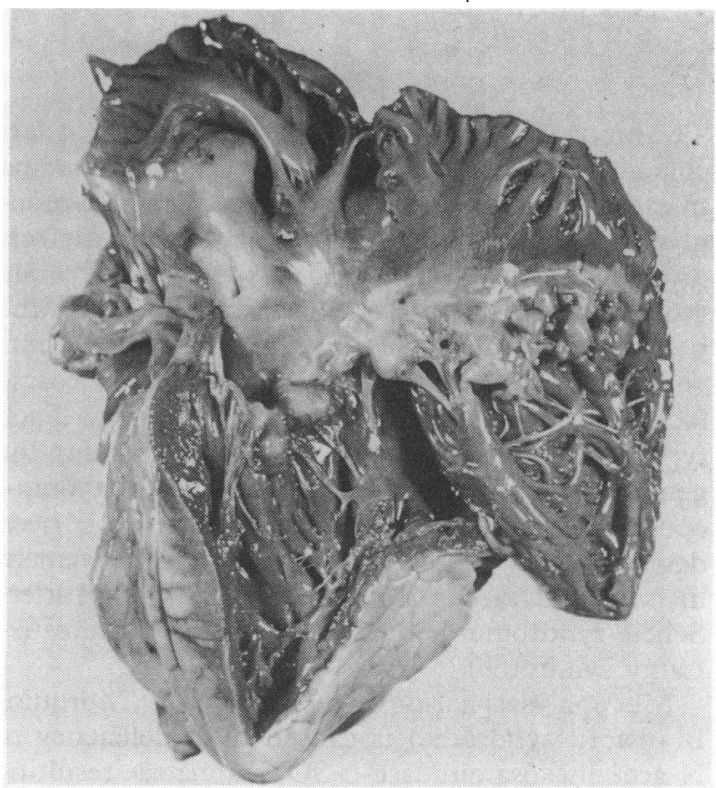

Fig. 2 The tricuspid valve cusps and chordae are thickened.

\section{Discussion}

Aortic valvular disease is well known to occur in mucopolysaccharidosis type IV. ${ }^{2}$ To our knowledge, mitral stenosis caused by infiltration of the valve cusps with mucopolysaccharide has not previously been reported in this syndrome and this appears to be the first case in which involvement of all four valves has been so clearly demonstrated.

Mitral stenosis caused by mucopolysaccharide infiltration of the valve cusps was first recorded in a case of "atypical gargoylism" by Vanace et al. ${ }^{5}$ At necropsy, infiltration of the mitral valve was demonstrated histochemically but it was not possible to characterise the disorder further. In 1975 Schieken et al. ${ }^{1}$ described two cases of mucopolysac- 
charidosis type I and one of type VI with mitral stenosis, the diagnosis being made on clinical and echocardiographic criteria, without necropsy data. It is interesting that in our patient no mitral diastolic murmur was heard, the diagnosis of mitral stenosis being first suggested on the echocardiogram and later confirmed at necropsy.

Patients with mucopolysaccharidosis type IV usually die before the age of 20 years and it has been suggested that aortic regurgitation is a late manifestation of this syndrome. ${ }^{2}$ Our patient survived until the age of $\mathbf{4 8}$ years without symptoms of valvular heart disease. From the necropsy it seems that mucopolysaccharide infiltration of all valves occurs in time, the symptoms and signs being dependent upon the site and severity of the predominant lesion(s). The usual cause of death is respiratory failure occurring after spinal cord compression resulting from skeletal deformity. Should these patients develop respiratory symptoms, the presence of valvular heart disease must be considered as an alternative cause for their deterioration.

We thank Professor W A Littler for helpful criticism, and Dr R M Cayton for permission to report this case.

\section{References}

1 Schieken RM, Kerber RE, Ionasescu VV, Zellweger $\mathrm{H}$. Cardiac manifestations of the mucopolysaccharidoses. Circulation 1975; 52: 700-5.

2 McKusick VA, Kaplan D, Wise D, et al. The genetic mucopolysaccharidoses. Medicine (Baltimore) 1965; 44: 445-83.

3 Lewis PW, Raine DN, Kennedy JF. Recognition of the mucopolysaccharidoses by 4 screening tests, including a refinement of the albumin turbidity test, and their differentiation by electrophoretic separation of urinary glycosaminoglycans. Ann Clin Biochem $1974 ; 11$ : 67-71.

4 Scott JE, Darling J. Differential staining of acid glycosaminoglycans (mucopolysaccharides) by Alcian blue in salt solutions. Histochemie 1965; 5: 221-33.

5 Vanace PW, Friedman S, Wagner BM. Mitral stenosis in an atypical case of gargoylism. Circulation 1960; 21 : 80-9.

Requests for reprints to $\operatorname{Dr} M$ A Ireland, Department of Cardiovascular Medicine, East Birmingham Hospital, Bordesley Green East, Birmingham B9 5ST. 